\title{
Use of CuO Particles as an Interface in LC-FTIR Analysis
}

\author{
Yan LI, ${ }^{* 1, * 2}$ Ran Guo, ${ }^{* 2, * 3}$ Shengnan Liu, ${ }^{* 2, * 4}$ Anqi He, ${ }^{2, * 5}$ Yanan BAO, ${ }^{2}, * 6$ Shifu Weng, ${ }^{* 2}$ \\ Yaping Huang, ${ }^{* 1 \dagger}$ Yizhuang $\mathrm{XU}_{\mathrm{U}},{ }^{2, * 5}$ Yukihiro OzAKI, ${ }^{* 2, * 7}$ Isao NodA, ${ }^{* 2, * 8}$ and Jinguang Wu ${ }^{* 2}$ \\ *1 Nanyang Institute of Technology, Nanyang 473000, P. R. China \\ *2 Beijing National Laboratory for Molecular Sciences, State Key Laboratory for Rare Earth Materials \\ Chemistry and Applications, College of Chemistry and Molecular Engineering, Peking University, \\ Beijing 100871, P. R. China \\ *3 College of Chemistry and Environmental Engineering, Shenzhen University, Shenzhen 518060, P. R. China \\ *4 College of Chemistry and Material Science, Hebei Normal University, Shijiazhuang 050024, P. R. China \\ *5 Ninghai Doubly Advanced Materials Co., Ltd., Ninghai 315602, P. R. China \\ *6 Liaoning Technical University, Fuxin 123000, P. R. China \\ *7 Department of Chemistry, School of Science, Kwansei Gakuin University, Sanda, Hyogo 669-1337, Japan \\ *8 Department of Materials Science and Engineering, University of Delaware, Newark, DE 19716, USA
}

\begin{abstract}
In this study, the feasibility of using copper oxide $(\mathrm{CuO})$ as an interface for the coupling of liquid chromatography (LC) and Fourier-transform infrared (FTIR) spectroscopy was investigated. $\mathrm{CuO}$ exhibits low absorption in the 4000 to $1000 \mathrm{~cm}^{-1}$ FTIR spectral region. In addition, LC-FTIR using $\mathrm{CuO}$ as the interface was extended to the analysis of sample mixtures containing benzamide and dioctyl sebacate; both analytes were successfully separated. After complete removal of the mobile phase, benzamide and dioctyl sebacate were successfully identified from the FTIR spectrum. Surprisingly, the interference from adsorbed water or conventionally used LC solvents in the FTIR spectrum was completely eliminated by using $\mathrm{CuO}$ particles as the interface in the off-line hyphenation of LC-FTIR. Based on these results, CuO demonstrates potential as an ideal interface for LC-FTIR analysis.
\end{abstract}

Keywords Copper oxide, interface, hyphenation, liquid chromatography, Fourier transform infrared spectroscopy

(Received September 12, 2016; Accepted November 4, 2016; Published January 10, 2017)

\section{Introduction}

Separation by liquid chromatography (LC) followed by spectroscopic identification has been extensively employed to analyze mixed samples in the fields of chemical, biological, and medical research and environmental protection, as well as in the food and pharmaceutical industries. ${ }^{1-4}$ Conventionally, LC is frequently coupled with UV-Vis detection for the identification of the separated substances based on their UV-Vis absorption. However, this method is not suitable for analytes that do not absorb in the UV-Vis region. Furthermore, UV-Vis spectra provide limited information regarding analyte structures. Hence, it is almost impossible to identify unknown substances based solely on their UV-Vis spectra. On the other hand, Fourier-transform infrared (FTIR) spectroscopy ${ }^{5,6}$ is a universal tool for identifying various organic compounds or metal complexes with organic ligands, even if these compounds do not absorb in the UV-Vis region. In addition, FTIR spectroscopy provides considerable information with respect to the functional groups of separated compounds. ${ }^{7-9}$ Furthermore, direct sample identification is also possible if a comprehensive FTIR database is available. Despite these aforementioned advantages of FTIR

$\dagger$ To whom correspondence should be addressed.

E-mail: yph_ny@163.com (Y. H.); xyz@pku.edu.cn (Y. X.) spectroscopy, the coupling of LC and FTIR has not been extensively reported. ${ }^{10,11}$ According to previous studies, two strategies have been utilized for hyphenating LC and FTIR.

1. On-line hyphenation: In this method, a flow cell is used for the continuous acquisition of spectra during experiments. ${ }^{12-14}$ That is, the FTIR spectrum of the eluate containing both the mobile phase and separated analytes is recorded. Although various approaches, e.g., chemometric methods, have been employed, interference from the mobile phase in the FTIR spectrum of an analyte still poses limitations for the extensive applications of on-line LC-FTIR analysis. ${ }^{15}$

2. Off-line hyphenation: In this method, an interface is adopted to collect samples eluted at different retention times. Then, the mobile phase is allowed to completely evaporate so that the FTIR spectra of the pure separated substances deposited on the interface can be recorded. ${ }^{16-25}$ Off-line hyphenation exhibits an advantage in that the interference from the mobile phase is completely eliminated, which in turn, improves the signal-to-noise ratio of the resultant FTIR spectra. Gagel et al. have utilized the highly IR reflective surface of metals, such as aluminum, as the interface for collecting separated substances from the eluate, followed by recording the corresponding FTIR spectra in the reflection mode. However, this approach is limited by issues of band distortion. ${ }^{22-24}$ A plausible approach is to use a hot germanium plate as the interface. During the experiment, the eluate obtained after LC separation, which elute 


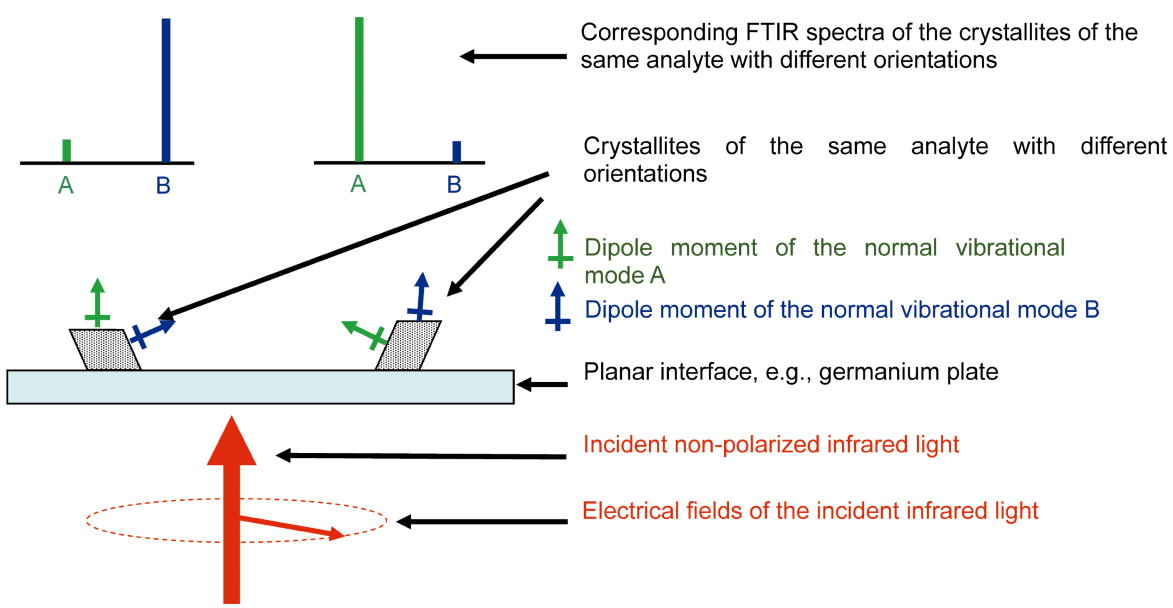

Scheme 1 Analytes crystallize on a planar interface. Because of the different orientation, different crystallites of the same substance may exhibit different FTIR spectra.
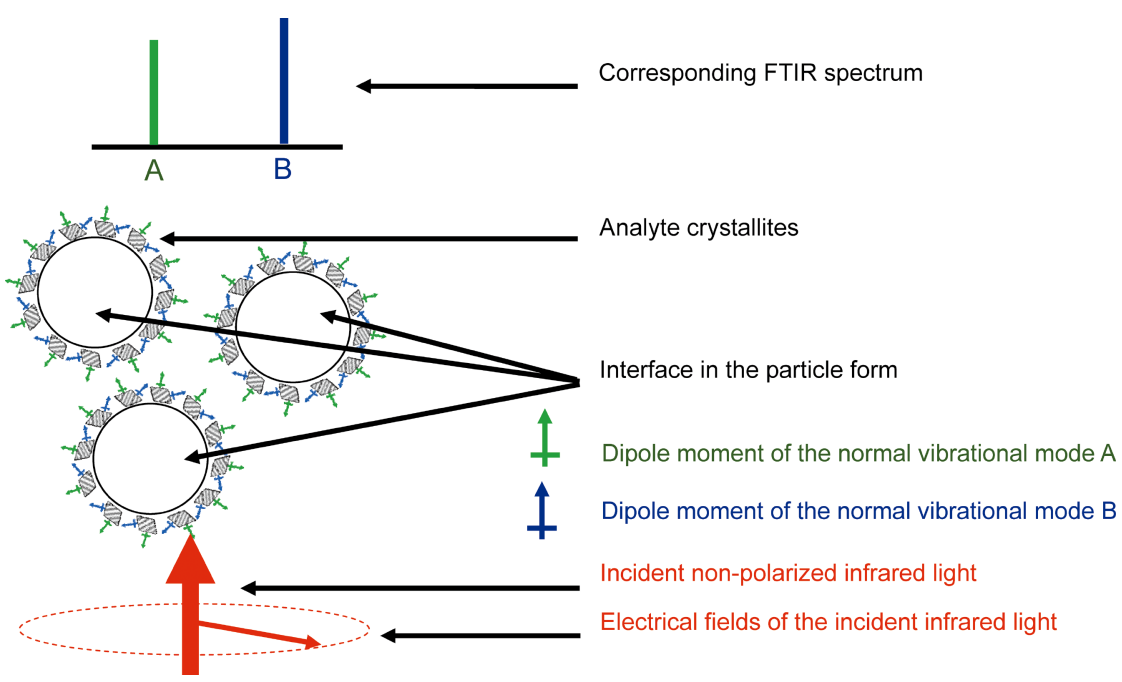

Scheme 2 Analytes crystallize on the interface in the particle form. The crystallites are isotropically oriented. The resultant FTIR spectra are free from the interference attributed to crystallite orientation.

at different retention times, are continuously deposited on the hot germanium plate for completely evaporating the mobile phase. Since germanium is transparent to IR light, the FTIR spectra of the separated samples can be recorded in the transmission mode. Nevertheless, a potential problem in utilizing this approach is associated with the orientation of separated substances (Scheme 1). After evaporation of the mobile phase, a separated analyte may crystallize on the germanium plate surface. Different crystalline planes or different lattice defects are exposed at different sites of the germanium plate, which can lead to different orientations of the crystallites of the separated samples. Hence, the obtained FTIR spectrum is dependent on the crystallite orientation. As a result, it is even possible that the FTIR spectrum of the same substance is different at different sites, which incurs additional difficulty for the chemical identification of separated unknown substances based on their FTIR spectra. An alternative to solving this issue is to use an interface in the powder form for the hyphenation of LC and FTIR. As shown in Scheme 2, the separated samples crystallize on the surface of each particle. In this case, the crystallites are isotropically orientated. Hence, the obtained
FTIR spectra are not dependent on the orientation.

For using an interface in the powder form, it is crucial to select a suitable substance as the interface. It should be transparent to IR as well as be insoluble. Previously, our group has conducted several studies for investigating stationary phases suitable for thin-layer chromatography (TLC)-FTIR analysis. ${ }^{7-9,26-31}$ A suitable stationary phase for use in TLC-FTIR should have the same prerequisites as the mentioned interface in powder form for HPLC-FTIR analysis. Although several insoluble inorganic salts, such as $\mathrm{BaF}_{2}, \mathrm{LaF}_{3}, \mathrm{AgI}, \mathrm{ZrO}_{2}$, and zinc oxide $(\mathrm{ZnO})$, are transparent to IR, a number of these salts tend to adsorb water. Unfortunately, it turns out to be almost impossible to remove the adsorbed water from these salts completely. As a result, strong and broad bands from $\mathrm{O}-\mathrm{H}$ stretching and $\mathrm{H}-\mathrm{O}-\mathrm{H}$ bending of adsorbed water bring about troublesome interference on the FTIR spectra in TLC-FTIR analysis. In several cases, the interference from adsorbed water makes it impossible to obtain FTIR spectra of analytes from the corresponding separated spots on TLC plates. The investigations give us an impression that inorganic salts that contain oxygen or fluorine tend to adsorb water via $\mathrm{O} \cdots \mathrm{H}-\mathrm{O}-\mathrm{H}$ or $\mathrm{F} \cdots \cdot \mathrm{H}-\mathrm{O}-\mathrm{H}$ 
hydrogen bonds.

Notably, we have found that copper oxide $(\mathrm{CuO})$ particles do not adsorb water despite the fact that $\mathrm{CuO}$ contains oxygen. From our further investigation, $\mathrm{CuO}$ powder was found to demonstrate potential for use as an ideal interface for LC-FTIR analysis.

\section{Experimental}

\section{Reagents}

HPLC-grade methanol was purchased from Dikma Technologies Inc. AR-grade dioctyl sebacate, ethanol, acetonitrile, $\mathrm{CuO}$, cerium dioxide $\left(\mathrm{CeO}_{2}\right)$, manganese dioxide $\left(\mathrm{MnO}_{2}\right), \mathrm{ZnO}$, and magnesium oxide $(\mathrm{MgO})$ were purchased from Beijing Chemical Company. Benzamide (98\% purity) was purchased from Aladdin Reagents. Silica gel (200 - 300 mesh) for LC was purchased from the Branch of Qingdao Haiyang Chemical Co., Ltd.

An LC column $(400 \times 15 \mathrm{~mm})$ was home-packed by natural sedimentation of silica gel in methanol under gravity.

\section{Instruments}

All of the FTIR spectra were recorded on an FTIR spectrometer (Thermo-Fisher Nicolet 6700) equipped with a diffuse reflectance accessory at a resolution of $4 \mathrm{~cm}^{-1}$, and a total of 32 scans were co-added to improve the signal-to-noise ratio. During the experiment, the FTIR spectrometer was purged with dry air to minimize any interference from water vapor. The Kubelka-Munk conversion was performed on all of the recorded FTIR spectra.

The samples were analyzed on an HPLC (Shimadzu) system, equipped with an LC-20AD pump, an injector mounted with a $20 \mu \mathrm{L}$ sample loop, and an SPD-M10A diode array detector. Separation was carried out on a Dikma C18 column (particle size: $5 \mu \mathrm{m}$, column dimensions: $150 \times 2.1 \mathrm{~mm}$ ) with methanol at a flow rate of $0.400 \mathrm{~mL} / \mathrm{min}$.

\section{Experimental procedure}

1. LC-FTIR analysis by using $\mathrm{CuO}$ as the interface. 1) Preparation of sample solutions. Sample 1: Benzamide $(0.50 \mathrm{~g})$ and dioctyl sebacate $(0.50 \mathrm{~g})$ were dissolved in methanol $(10.0 \mathrm{~mL})$, affording a mixed sample. Sample 2: Dioctyl sebacate $(0.50 \mathrm{~g})$ was dissolved in methanol $(10.0 \mathrm{~mL})$, affording a dioctyl sebacate solution. Sample 3: Benzamide $(0.50 \mathrm{~g})$ was dissolved in methanol $(10.0 \mathrm{~mL})$, affording a benzamide solution.

2) LC separation. First, $0.60 \mathrm{~mL}$ of Sample 1 was loaded on a home-packed LC column with methanol as the mobile phase. Elution was carried out under gravity, and the eluate was collected every $30 \mathrm{~s}$.

3) Approximately $0.05 \mathrm{~mL}$ of each eluted sample was dropped into a cylindrical slot $(12 \times 3 \mathrm{~mm})$ of a diffuse reflectance accessory filled with $\mathrm{CuO}$ powder. After the solvent was completely vaporized, the FTIR spectrum of each sample was recorded.

4) A three-dimensional (3D) chromatogram was constructed to illustrate the elution profile of the benzamide/dioctyl sebacate mixed sample after LC separation, generated using a program written in our laboratory with MATLAB (The MathWorks Inc.). For comparison, samples 2 and 3 were analyzed in the same manner so as to ensure the appropriate detection of benzamide and dioctyl sebacate from the FTIR spectra by LC-FTIR analysis with $\mathrm{CuO}$ powder as the interface.

2. Preliminary HPLC-FTIR analysis using $\mathrm{CuO}$ as the interface. 1) Preparation of sample solutions. Sample 4: Benzamide

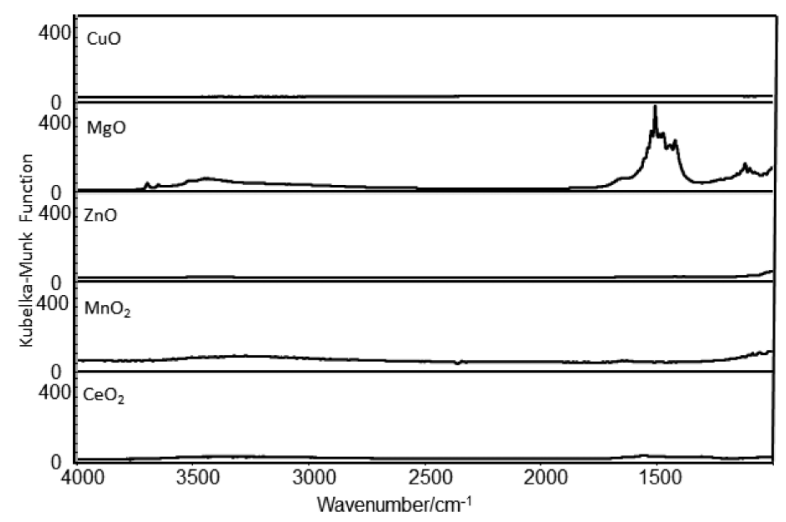

Fig. 1 FTIR spectrum of $\mathrm{CuO}$ particles recorded in the diffuse reflection mode. For comparison, FTIR spectra of $\mathrm{MgO}, \mathrm{ZnO}, \mathrm{MnO}_{2}$ and $\mathrm{CeO}_{2}$ in the diffuse reflection mode are also shown.

$(0.192 \mathrm{~g})$ and dioctyl sebacate $(0.210 \mathrm{~g})$ were dissolved in methanol $(10.0 \mathrm{~mL})$, affording a mixed sample. Sample 5: Benzamide $(0.192 \mathrm{~g})$ was dissolved in methanol $(10.0 \mathrm{~mL})$, affording a benzamide solution. Sample 6: Dioctyl sebacate $(0.210 \mathrm{~g})$ was dissolved in methanol $(10.0 \mathrm{~mL})$, affording a dioctyl sebacate solution.

2) HPLC separation. First, $20.0 \mu \mathrm{L}$ of sample 4 was loaded on the HPLC system with methanol as the mobile phase at a flow rate of $0.400 \mathrm{~mL} / \mathrm{min}$. The eluted solution was collected every $30 \mathrm{~s}$.

3) Approximately $0.05 \mathrm{~mL}$ of the eluted sample was dropped into a cylindrical slot $(12 \times 3 \mathrm{~mm})$ of a diffuse reflectance accessory filled with $\mathrm{CuO}$ powder. After the solvent was completely evaporated, the FTIR spectrum was recorded.

For comparison, samples 5 and 6 were analyzed in the same manner so as to ensure appropriate detection of benzamide and dioctyl sebacate from the FTIR spectra by HPLC-FTIR analysis using $\mathrm{CuO}$ powder as the interface.

\section{Results and Discussion}

Figure 1 shows the FTIR spectrum of $\mathrm{CuO}$ particles (top panel). For comparison, the FTIR spectra of other metal oxides are also shown. In the FTIR spectra of $\mathrm{MgO}, \mathrm{CeO}_{2}, \mathrm{MnO}_{2}$, and $\mathrm{ZnO}$, absorption bands are observed in the $3600-3000 \mathrm{~cm}^{-1}$ region, attributed to the $\mathrm{O}-\mathrm{H}$ stretching modes of adsorbed water, and an absorption band is observed in the $1500-1300 \mathrm{~cm}^{-1}$ region, attributed to the antisymmetric $\mathrm{O}-\mathrm{C}=\mathrm{O}$ stretching mode of carbonate. The results indicated that adsorbed water or carbonate are present in these metal oxides. On the other hand, an absorption band is not observed in $4000-1000 \mathrm{~cm}^{-1}$ region in the FTIR spectrum of $\mathrm{CuO}$, indicating that $\mathrm{CuO}$ is transparent in the mid-IR region. Moreover, adsorbed water is not observed on the $\mathrm{CuO}$ particle surface even if $\mathrm{CuO}$ is exposed to air for a long time.

For examining whether the mobile phase is completely removed from the $\mathrm{CuO}$ particle surface, the following experiments were performed. Water $(0.05 \mathrm{~mL})$, methanol $(0.05 \mathrm{~mL})$, ethanol $(0.05 \mathrm{~mL})$, and acetonitrile $(0.05 \mathrm{~mL})$ were applied on $\mathrm{CuO}$ powder, and then the FTIR spectra of $\mathrm{CuO}$ / methanol, $\mathrm{CuO} /$ ethanol, $\mathrm{CuO} /$ acetonitrile, and $\mathrm{CuO} /$ water mixtures were recorded. The corresponding FTIR spectra are shown as trace 1 in Figs. 2(A) - 2(D). Strong absorption peaks for water, methanol, ethanol, and acetonitrile are clearly 
(A)

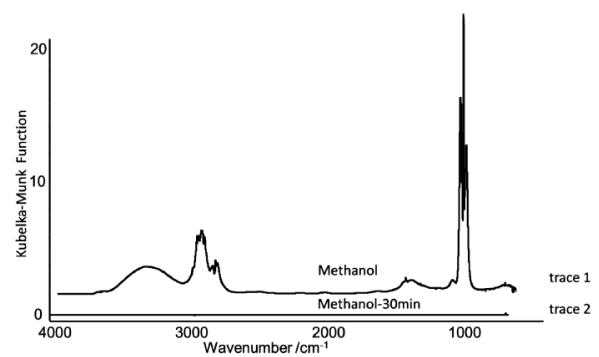

(C)

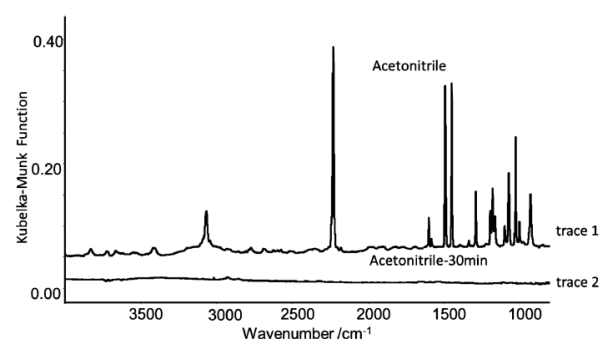

(B)

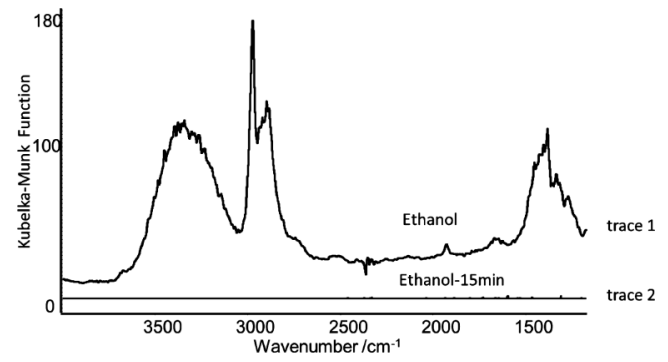

(D)

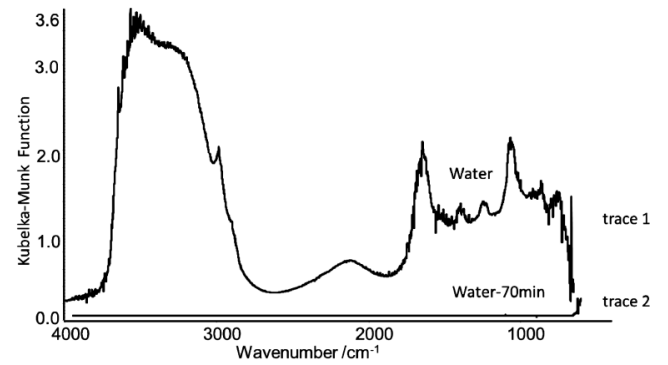

Fig. 2 (A) Trace 1: FTIR spectrum of $\mathrm{CuO}$ particles in the diffuse reflection mode after the deposition of $0.05 \mathrm{~mL}$ methanol. Trace 2: FTIR spectrum of $\mathrm{CuO}$ in the diffuse reflection mode after the $\mathrm{CuO} /$ methanol mixture was placed in air at room temperature for $15 \mathrm{~min}$. (B) Trace 1: FTIR spectrum of $\mathrm{CuO}$ particles in the diffuse reflection mode after the deposition of $0.05 \mathrm{~mL}$ of ethanol. Trace 2: FTIR spectrum of $\mathrm{CuO}$ in the diffuse reflection mode after the $\mathrm{CuO} /$ ethanol mixture was placed in air at room temperature for $15 \mathrm{~min}$. (C) Trace 1: FTIR spectrum of $\mathrm{CuO}$ particles in the diffuse reflection mode after the deposition of $0.05 \mathrm{~mL}$ of acetonitrile. Trace 2: FTIR spectrum of $\mathrm{CuO}$ in the diffuse reflection mode after the $\mathrm{CuO}$ / acetonitrile mixture was placed in air at room temperature for $30 \mathrm{~min}$. (D) Trace 1: FTIR spectrum of $\mathrm{CuO}$ particles in the diffuse reflection mode after the deposition of $0.05 \mathrm{~mL}$ of water. Trace 2: FTIR spectrum of $\mathrm{CuO}$ in the diffuse reflection mode after the $\mathrm{CuO} /$ water mixture was placed in air at room temperature for $70 \mathrm{~min}$.

observed in all of these FTIR spectra. Next, the mixtures were placed in air at room temperature for different periods of time (the exact time for different solvent can be found in the caption of Fig. 2), and then the FTIR spectra of the four $\mathrm{CuO}$ samples were recorded. The corresponding FTIR spectra are shown as trace 2 in Figs. 2(A) - 2(D). Absorption peaks corresponding to water, methanol, ethanol, and acetonitrile are not observable any more, clearly indicative of no adsorption or weak adsorption of water, methanol, ethanol. The results clearly demonstrate that $\mathrm{CuO}$ particles exhibit weak or no adsorption of water, methanol, ethanol and acetonitrile. Hence, $\mathrm{CuO}$ demonstrates potential for use as an ideal interface for LC-FTIR analysis.

For investigating whether $\mathrm{CuO}$ can be applied to an actual compound analysis, we use the LC-FTIR method by using $\mathrm{CuO}$ as an interface to analyze benzamide/dioctylsebacate (sample 1). Notably, benzamide and dioctylsebacate are both colorless compounds; thus, the elution profile of benzamide or dioctylsebacate cannot be reflected by the variation of the color of the eluate. Nevertheless, the elution profile after LC separation can be revealed by the FTIR spectra. In this study, the eluted samples were sequentially collected. Each eluted sample was dropped into a slot filled by $\mathrm{CuO}$ particles. After completely evaporating the mobile phase by exposing the mixture to air at room temperature for $30 \mathrm{~min}$, an FTIR spectrum of each sample was recorded in the diffuse reflection mode. The FTIR spectra thus obtained were organized according to the order of the sample retention times to construct a 3D chromatogram (the first dimension is the wavenumber from an FTIR spectrum, the second dimension is the retention time, and the third dimension is the value obtained from the Kubelka-Munk function of the corresponding FTIR spectrum measured in the diffuse reflection mode). The $3 \mathrm{D}$ chromatogram is shown as a

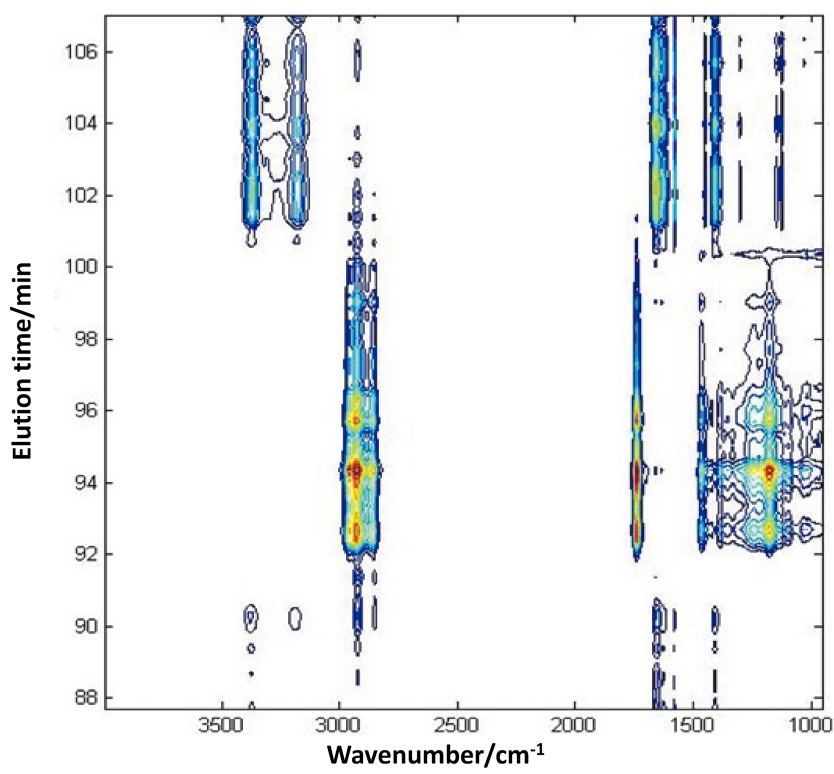

Fig. 3 Three-dimensional chromatogram obtained from the LC/FTIR analysis of a mixture of benzamide and dioctyl sebacate by using $\mathrm{CuO}$ as the interface.

contour plot (Fig. 3). From the 3D chromatogram, two separated compounds are identified. The retention times for the first and second compounds are 92.0-100.4 and 101.0-107.0 min, respectively. The absence of an absorption peak in the $3600-3000 \mathrm{~cm}^{-1}$ region for the first compound excludes the 


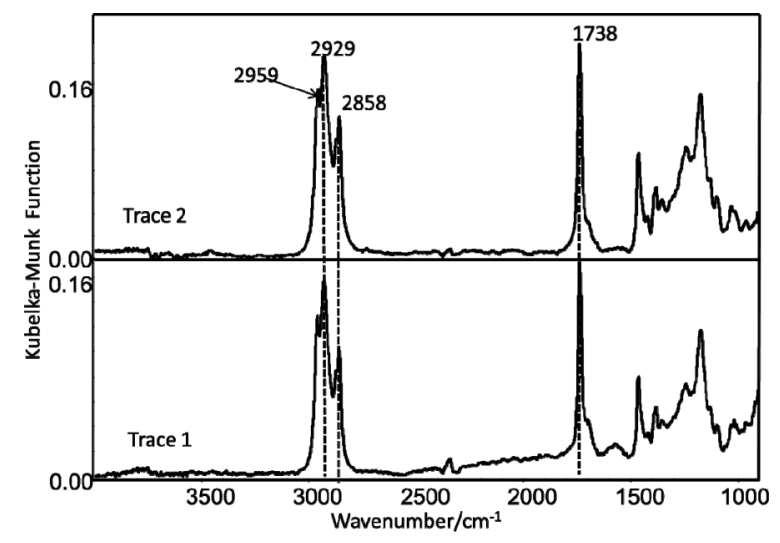

Fig. 4 Trace 1: FTIR spectrum of an eluted sample in the diffuse reflection mode; its elution time is between 92.7 and $93.0 \mathrm{~min}$ after the LC separation of sample 1. Trace 2: FTIR spectrum of dioctyl sebacate in the diffuse reflection mode obtained from the eluate from sample 2 after LC separation.

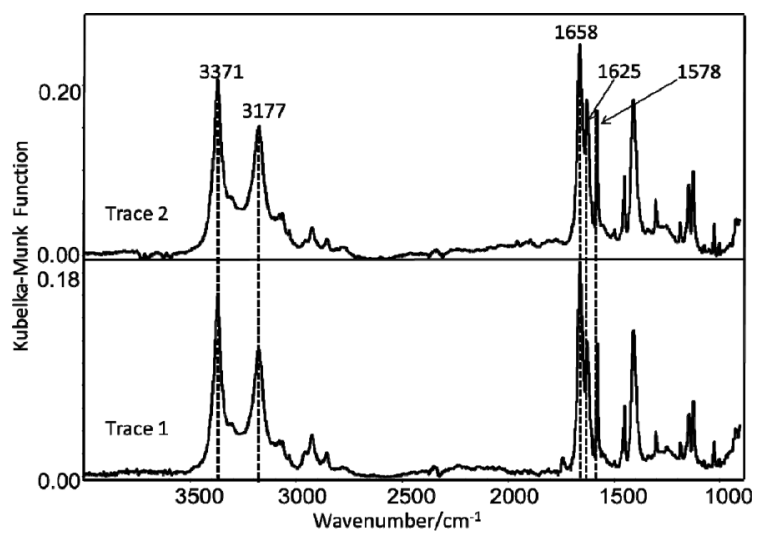

Fig. 5 Trace 1: FTIR spectrum of an eluted sample in the diffuse reflection mode; its elution time is between 101.7 and $102.0 \mathrm{~min}$ after the LC separation of sample 1. Trace 2: FTIR spectrum of benzamide in the diffuse reflection mode obtained from the eluted sample 3 after LC separation.

possibility that the first compound is benzamide. Hence, the first compound is dioctylsebacate. From the $3 \mathrm{D}$ chromatogram, an FTIR spectrum of the first compound with a retention time of 92.7-93.0 min is selected and shown as trace 1 in Fig. 4. Strong C-H stretching bands and a strong carbonyl band at 2929 and $1738 \mathrm{~cm}^{-1}$, respectively, are observed for the first compound, indicative of the first compound is dioctylsebacate. For comparison, the methanol solution of dioctylsebacate (sample 2) was also analyzed by LC-FTIR.

Figure 4 (trace 2) shows a typical FTIR spectrum of dioctyl sebacate. The spectrum shown in trace 1 is almost the same as that shown in trace 2, confirming that the first compound is dioctylsebacate. For the second compound, a typical FTIR spectrum of the eluted sample with a retention time of 101.7 - $102.0 \mathrm{~min}$ is shown as trace 1 in Fig. 5. In the spectrum, two strong peaks are observed at 3371 and $3177 \mathrm{~cm}^{-1}$ for the second compound, possibly attributed to the N-H stretching vibrations. Moreover, two carbonyl bands are also observed at 1659 and $1625 \mathrm{~cm}^{-1}$, respectively. In addition, a sharp band corresponding to the $\mathrm{C}=\mathrm{C}$ stretching vibration is observed at around $1578 \mathrm{~cm}^{-1}$. These bands are the characteristic bands of benzamide. For confirming whether the second compound is

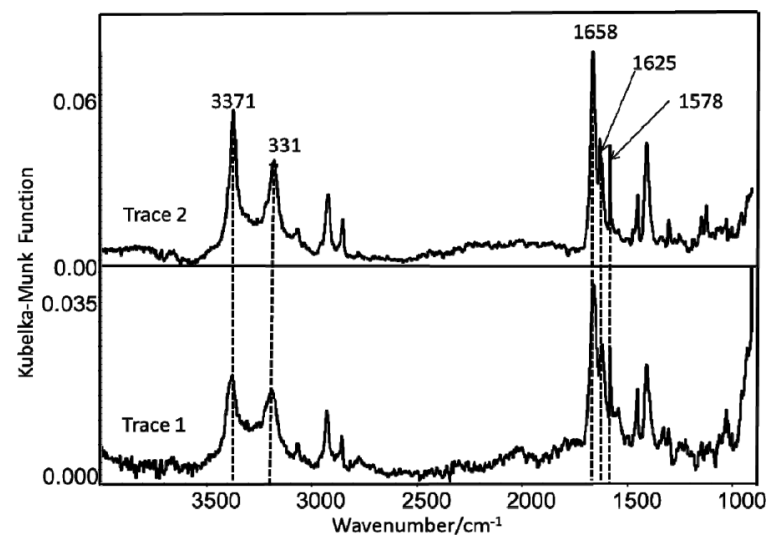

Fig. 6 Trace 1: FTIR spectrum of an eluted sample in the diffuse reflection mode; its elution time is between 1.5 and $2.5 \mathrm{~min}$ after the HPLC separation of sample 4. Trace 2: FTIR spectrum of benzamide in the diffuse reflection mode obtained from the eluted sample 5 after HPLC separation.

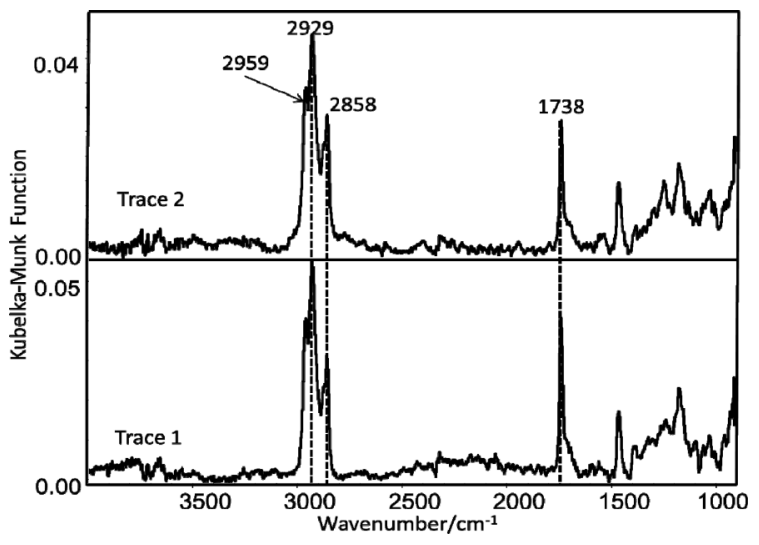

Fig. 7 Trace 1: FTIR spectrum of an eluted sample in the diffuse reflection mode; its elution time is between 3.0 and $4.0 \mathrm{~min}$ after the HPLC separation of sample 4. Trace 2: FTIR spectrum of dioctyl sebacate in the diffuse reflection mode obtained from the eluted sample 6 after HPLC separation.

benzamide, a methanol solution of benzamide (sample 3 ) is also analyzed by the LC-FTIR method. A typical FTIR spectrum is shown as trace 2 in Fig. 5. The spectrum of trace 1 in Fig. 5 is almost identical to that of trace 2 in Fig. 5, indicating that the second compound is benzamide.

The above results demonstrate that the hyphenation of LC and FTIR using $\mathrm{CuO}$ as the interface is applicable in real chemical system.

For rendering LC-FTIR analysis using $\mathrm{CuO}$ as the interface for extensive applications in analytical chemistry, it is indispensable to realize the hyphenation of HPLC and FTIR. Thus, a preliminary investigation is performed using HPLC-FTIR analysis. A challenge of HPLC-FTIR analysis is that both the amount of the samples and the concentrations of the analytes are significantly reduced in comparison with the LC-FTIR experiment.

In this study, a mixture containing benzamide and dioctylsebacate was first separated by HPLC. The eluted peak corresponding to benzamide was identified by its absorbance at $254 \mathrm{~nm}$ using a diode array detector, while that corresponding to dioctylsebacate was identified by its absorbance at $190 \mathrm{~nm}$. 
The HPLC results demonstrate that the two compounds are completely separated. The corresponding eluted sample was collected, and its FTIR spectrum was recorded. The obtained FTIR spectra of benzamide and dioctylsebacate are shown as trace 1 in Figs. 6 and 7, respectively. Although the intensities of the peaks of benzamide and dioctylsebacate in the FTIR spectra after HPLC separation are significantly reduced, characteristic bands of benzamide and dioctyl sebacate are still clearly observed. For comparison, the methanol solution containing only benzamide (sample 5) and only dioctyl sebacate (sample 6) are also analyzed by HPLC-FTIR. The corresponding FTIR spectra are shown as trace 2 in Figs. 6 and 7. The spectrum of trace 1 in Fig. 6 is similar to that of trace 2. A similar result can be observed in Fig. 7. The above results indicated that HPLC-FTIR by using $\mathrm{CuO}$ as the interface can be employed for analyzing an actual mixture. Currently, studies for improving the sensitivity of HPLC-FTIR analysis by using $\mathrm{CuO}$ as the interface are underway in our laboratory.

\section{Conclusions}

Despite significant potential, the hyphenation or coupling of LC and FTIR has not been extensively applied for practical analysis. A suitable interface is crucial for hyphenation. A suitable stationary phase for LC-FTIR should have prerequisites of IR transparency and particle insolubility. Although several insoluble inorganic salts (e.g., $\mathrm{BaF}_{2}, \mathrm{LaF}_{3}, \mathrm{AgI}, \mathrm{ZrO}_{2}, \mathrm{ZnO}$ ) are transparent to IR, a number of these salts tend to adsorb a considerable amount of water, which is almost impossible to remove. In this study, $\mathrm{CuO}$ was found to be effective as an interface for LC-FTIR analysis, attributed to its excellent transparency to IR light. In addition, the interference from adsorbed water or conventionally used LC solvents in the FTIR spectrum was completely eliminated by using $\mathrm{CuO}$ particles as the interface in off-line LC-FTIR hyphenation. Satisfactory results were obtained for the off-line LC-FTIR analysis of mixtures by using $\mathrm{CuO}$ as the interface. These encouraging results indicated that a CuO-based off-line LC-FTIR hyphenation approach demonstrates immense potential for analyzing various systems.

\section{Acknowledgements}

This study was financially supported by the National Natural Science Foundation of China (51373003) and the Excellent Young Academic Fund of NYIST (50104048).

\section{References}

1. M. Khalil, N. Demirdoven, and A. Tokmakoff, J. Phys. Chem. A, 2003, 107, 5258.

2. A. Ajayaghosh, Acc. Chem. Res., 2005, 38, 449.

3. D. W. Lupo and M. Quack, Chem. Rev., 1987, 87, 181.

4. Y. S. Kim and R. M. Hochstrasser, J. Phys. Chem. B, 2009, 113,8231 .

5. J. G. Wu, "Modern FTIR Spectroscopy, Technology and Application", 1994, Science and Technology Press, Beijing.

6. S. F. Weng, "Fourier Transform Infrared Spectrometer",
2010, Chemical Industry Press, Beijing.

7. Q. Zhu, H. J. Wu, F. Wang, A. Q. He, K. Huang, Y. J. Wei, C. G. Liu, Y. J. Zhai, S. F. Weng, Z. L. Yang, Y. Z. Xu, I. Noda, and J. G. Wu, J. Planar Chromatogr-Mod. TLC, 2014, 27, 80 .

8. X. K. Fan, R. Guo, H. J. Wu, J. J. Shi, A. Q. He, Y. J. Wei, C. G. Liu, S. F. Weng, Z. L. Yang, Y. Z. Xu, I. Noda, and J. G. Wu, J. Spectrosc., 2013, Article ID 976360.

9. J. Liu, F. S. Zhou, R. Guo, Y. Jiang, X. K. Fan, A. Q. He, Y. J. Zhai, S. F. Weng, Z. L. Yang, Y. Z. Xu, I. Noda, and J. G. Wu, J. Spectrosc., 2014, Article ID 925705.

10. S. Armenta, S. Garrigues, and M. de la Guardia, $\operatorname{Tr} A C$, Trends Anal. Chem., 2007, 26, 775.

11. M. Gallignani and M. R. Brunetto, Talanta, 2004, 64, 1127.

12. B. M. Patterson, N. D. Danielson, and A. J. Sommer, Anal. Chim. Acta, 2007, 583, 231.

13. K. Istvan, G. Keresztury, and J. Fekete, J. Liq. Chromatogr. Relat. Technol., 2005, 28, 407.

14. R. Schulte-Ladbeck, A. Edelmann, G. Quintas, B. Lendl, and U. Karst, Anal. Chem., 2006, 78, 8150.

15. G. W. Somsen, C. Gooijer, and U. A. T. Brinkman, J. Chromatogr. A, 1999, 856, 213.

16. R. A. Todebush, L. T. He, and J. A. de Haseth, Anal. Chem., 2003, 75, 1393.

17. M. A. Mottaleb and D. Littlejohn, Anal. Sci., 2001, 17, 429.

18. K. Torabi, A. Karami, S. T. Balke, and T. C. Schunk, J. Chromatogr. A, 2001, 910, 19.

19. S. J. Kok, N. C. Arentsen, P. J. C. H. Cools, Th. Hankemeier, and P. J. Schoenmakers, J. Chromatogr. A, 2002, 948, 257.

20. N. D. Danielson, J. E. Katon, S. P. Bouffard, and Z. H. Zhu, Anal. Chem., 1992, 64, 2183.

21. S. P. Bouffard, J. E. Katon, A. J. Sommer, and N. D. Danielson, Anal. Chem., 1994, 66, 1937.

22. J. J. Gagel and K. Biemann, Anal. Chem., 1987, 59, 1266.

23. G. W. Somsen, R. J. van de Nesse, C. Gooijer, U. A. T. Brinkman, N. H. Velthorst, T. Visser, P. R. Kootstra, and A. P. J. M. de Jong, J. Chromatogr., 1991, 552, 635.

24. R. Fuoco, S. L. Pentoney Jr., and P. R. Griffiths, Anal. Chem., 1989, 61, 2212.

25. M. A. Castles, L. V. Azarraga, and L. A. Carreira, Appl. Spectrosc., 1986, 40, 673.

26. X. Liu, Q. H. Pan, J. Ding, Q. Zhu, A. Q. He, S. J. Yue, X. P. Li, L. P. Hu, J. M. Xia, C. G. Liu, Y. J. Wei, J. Yu, Z. L. Yang, X. Zhu, Y. Z. Xu, and J. G. Wu, Spectrosc. Spect. Anal., 2011, 31, 1767.

27. Q. Zhu, X. Su, H. J. Wu, Y. J. Zhai, J. M. Xia, Y. Z. Xu, and J. G. Wu, Spectrosc. Spect. Anal., 2012, 32, 1790.

28. W. Liu, H. J. Wu, X. P. Wang, Q. Zhu, T. G. Kang, A. Q. He, S. F. Weng, Z. L. Yang, J. M. Xia, Y. Z. Xu, J. G. Wu, Z. F. Song, S. F. Weng, Z. L. Yang, Y. Z. Xu, and J. G. Wu, Chem. J. Chin. Univ., 2013, 34, 1347.

29. X. K. Fan, Y. Jiang, J. J. Shi, C. G. Liu, Y. J. Wei, Z. F. Song, S. F. Weng, Z. L. Yang, Y. Z. Xu, and J. G. Wu, Chem. J. Chin. Univ., 2014, 35, 741.

30. F. Wang, H. J. Wu, Q. Zhu, K. Huang, Y. J. Wei, C. G. Liu, Y. J. Zhai, Z. L. Yang, S. F. Weng, Y. Z. Xu, I. Noda, and J. G. Wu, Anal. Methods, 2013, 5, 4138.

31. Y. Jiang, X. Y. Kang, D. Q. Gao, A. Q. He, R. Guo, X. K. Fan, Y. J. Zhai, J. M. Xia, Y. Z. Xu, I. Noda, and J. G. Wu, RSC Adv., 2015, 5, 21544. 